\title{
DESEMPENHO, RENDIMENTO DE CARCAÇA E COMPORTAMENTO DE FRANGOS DE CORTE CRIADOS EM CAMA DE MARAVALHA OU AREIA
}

Lilian Francisco Arantes de Souza, Nadiele Taise Massaranduba, Isadora de Almeida Ruiz, Adriele de Souza Gomes, Ana Paula Souza Costa, Ana Flávia Grillo Silva

Universidade do Oeste Paulista - UNOESTE, Curso de Medicina Veterinária, Presidente Prudente, SP. E-mail: lilian@unoeste.br

\section{RESUMO}

Esta pesquisa teve como objetivo avaliar o desempenho, comportamento e características da carcaça de frangos de corte criados em cama de maravalha ou areia. 120 pintos foram distribuídos em 2 tratamentos com 6 repetições. Os dois tipos de cama avaliados não influenciaram o desempenho das aves. A criação em cama de areia resultou em maior frequência de comportamentos ingestivos, exploratórios e agressivos e menor de repouso, menores frequências de problemas locomotores, maior rendimento de peito, maior peso relativo de fígado, moela e intestino delgado em comparação á cama de maravalha. Concluiu-se que cama de areia é uma alternativa viável para substituição da cama de maravalha e aves criadas em cama de areia são mais ativas.

Palavras-chave: bem-estar animal; etograma; locomoção; sistema digestório; tipos de cama.

\section{PERFORMANCE, CARCASS YIELD AND BEHAVIOR OF BROILERS REARED ON WOOD SHAVINGS OR SAND BED}

\begin{abstract}
This research aimed to evaluate the performance, behavior and carcass characteristics of broiler chickens raised in wood shavings or sand bedding. 120 chicks were distributed in two treatments with 6 repetitions. The two bedding types evaluated did not affect the performance of the birds. Creating sand bedding resulted in higher frequency of ingestive, exploratory and aggressive behaviors and less rest, lower frequencies of locomotor problems, higher breast yield, increased relative weight of liver, gizzard and small intestine in comparison will shavings bedding. It was concluded that the sand bedding is a viable alternative to replace the wood shavings bedding and birds reared in sand bedding are more active.
\end{abstract}

Keywords: animal welfare; bedding types; digestive system; ethogram, locomotion.

\section{INTRODUÇÃO}

A cama de aviário é composta por materiais utilizados como substratos para forração do piso, juntamente com excretas, descamações da pele, penas, ração, entre outros. Os objetivos dos substratos são amortecer impactos, absorver a umidade, além de isolamento térmico (PAGANINI; MENDES, 2004). No Brasil, são utilizados materiais como maravalha, casca de arroz, amendoim, café, capins picados e palhadas de culturas (AVILA et al., 2008; CARVALHO et al., 2011; NEME et al., 2000). Entretanto, pesquisas mostram a possibilidade de utilização de materiais alternativos como areia, vermiculita e produtos do papel (BILGILI et al., 1999a,b; LIEN et al., 1992; MILES et al., 2011).

A utilização de areia como material para cama de aviário tem despertado o interesse de pesquisadores, uma vez que permite melhores condições relacionadas ao bem-estar animal (BILGILI et al., 2009), além da indisponibilidade e alto custo de outros materiais. Pesquisas comparando maravalha, e areia mostram resultados positivos tanto para desempenho como para qualidade da carcaça (BILGILI et al., 1999a,b). Por outro lado, Anisuzzaman e Chowdhury (1996) compararam diferentes tipos de material, inclusive areia, e constataram que frangos criados em cama de 
casca de arroz apresentaram melhor desempenho em relação aos demais materiais.

O melhoramento genético dos frangos de corte resultou em aumento expressivo do peso corporal. Havenstein et al. (2013) mostraram que, aos 42 dias, frangos melhorados geneticamente apresentam peso 4,5 vezes superior a frangos com genética estabilizada. Corr et al. (2003) concluíram que frangos melhorados geneticamente apresentam grandes modificações na marcha, mostrando dificuldades para caminhar. Ainda, aves melhoradas geneticamente apresentam grande inatividade comportamental (WEEKS et al., 2000). Nesse sentido, medidas estão sendo implementadas para estimular a movimentação dos frangos de corte como dificultar o acesso dos animais aos bebedouros e comedouros (BIZERAY et al., 2002), utilização de programas de luz (BUYSE et al., 1996) e uso da areia como material para cama de aviário (SHIELDS et al., 2005).

O objetivo desse trabalho foi avaliar o desempenho, comportamento, rendimento de carcaça e cortes comerciais, peso relativo de vísceras comestíveis e órgãos do sistema digestório de frangos de corte aos 42 dias de idade criados em cama de maravalha ou areia.

\section{METODOLOGIA}

O experimento foi realizado no Aviário Experimental da UNOESTE. O trabalho foi aprovado pelo Comitê de Ética no Uso de Animais (CEUA) da UNOESTE (Protocolo 323). Foram utilizados 120 pintos da linhagem Cobb, machos, distribuídos em delineamento inteiramente casualizado em 2 tratamentos (Maravalha e Areia) com 6 repetições de 10 aves cada. As aves receberam manejo convencional, ração balanceada (ROSTAGNO et al., 2011) e água ad libitum. Foram utilizados maravalha de pinus e areia grossa lavada. A altura das camas foi de 12 e $5 \mathrm{~cm}$ para maravalha e areia, respectivamente. As camas foram reviradas semanalmente para evitar a compactação.

O desempenho foi avaliado aos 42 dias por meio do consumo de ração, ganho de peso, conversão alimentar, viabilidade criatória e índice de eficiência produtiva. O comportamento foi avaliado, aos 35 dias, por meio do etograma apresentado na Tabela 1, de acordo com Bonamigo et al. (2011) e por meio do agrupamento de atividades (Tabela 2) segundo Picoli (2004). Em cada avaliação foi selecionada uma parcela experimental (10 aves) de cada tratamento de forma aleatória. As aves foram avaliadas visualmente a cada 30 minutos por um período de 12 horas (6h00 às 18h00).

Tabela 1 - Etograma desenvolvido para avaliação comportamental dos frangos de corte criados em cama de maravalha ou areia.

\begin{tabular}{lr}
\hline Atividade & Definição \\
\hline Acocorado & Ave está sentada ou deitada sem qualquer outra atividade \\
Trocando de posição & Ave está em movimento, alternado entre posições \\
Comendo & Ave está se alimentando, independente de estar em pé ou sentada \\
Bebendo & Ave está bebendo água, independente se estas em pé ou sentada \\
Em pé & Quando a ave está em pé ou caminhando \\
Limpando penas & Quando a ave alinha suas penas com o bico \\
\hline
\end{tabular}

Tabela 2 - Agrupamento de comportamentos desenvolvido para avaliação comportamental dos frangos de corte criados em cama de maravalha ou areia.

\begin{tabular}{|c|c|}
\hline $\begin{array}{l}\text { Comportamento } \\
\text { agrupado }\end{array}$ & Definição \\
\hline Ingestivo & Ave se alimentando ou bebendo água \\
\hline Repouso & Ave parada ou deitada \\
\hline Exploratório & Ave em movimento, andando ou ciscando \\
\hline Conforto & Ave esticando membros, banho de poeira ou limpando penas \\
\hline Agressivo & Ave bicando ou em interação agonística* \\
\hline
\end{tabular}

Aos 35 dias de idade, duas aves por parcela experimental foram selecionadas aleatoriamente para avaliação da habilidade de locomoção, sendo utilizado o método de escore 
de andadura, descrito por Jones et al. (2005). As aves caminharam por, no mínimo, 10 passos e foram classificadas de acordo com a dificuldade de locomoção, dividida em três níveis: zero para o frango sem problemas locomotores; um para aves caminhando de forma irregular, descompassadas e desbalanceadas; e dois para as aves com relutância para se mover, incapazes de caminhar muitos passos antes de se sentar.

Aos 42 dias de idade foi realizada a retirada de dois frangos por unidade experimental. Após jejum de alimento de 12 horas, os frangos foram insensibilizados por deslocamento cervical (Diretrizes da prática de eutanásia do CONCEA) e abatidos por sangria mediante corte da veia jugular. Os cortes de peito, de coxas, sobrecoxas, asas e gordura abdominal foram feitos por uma única pessoa treinada. Os rendimentos de carcaça e de corte comerciais foram expressos em relação ao peso corporal no momento do abate. Foi considerada carcaça a ave livre de cabeça, pescoço, pés, vísceras e penas. As vísceras comestíveis (fígado, moela e coração) e os componentes do sistema digestório também foram pesados e seu peso relativo calculado em relação ao peso corporal da ave viva aos 42 dias.

Os dados referentes ao desempenho, características da carcaça e peso relativo de órgãos do sistema digestório foram analisados por meio do teste $t$ de Student. A análise de comportamento e habilidade de locomoção foram realizadas por meio do teste qui-quadrado. As análises foram realizadas no programa BioEstat 5.3 utilizando nível de significância de $5 \%(p<0,05)$.

\section{RESULTADOS}

Não houve diferença significativa $(p>0,05)$ entre os dois tipos de cama avaliados sobre nenhum parâmetro de desempenho (Tabela 3).

A Tabela 4 mostra os resultados referentes ao comportamento avaliado por meio de etograma e agrupado.

Tabela 3 - Valores médios de consumo de ração (g), ganho de peso (g), conversão alimentar (g/g), viabilidade criatória (\%) e índice de eficiência produtiva (pontos) de frangos de corte aos 42 dias criados em cama de maravalha e areia.

\begin{tabular}{lccc}
\hline Desempenho & Maravalha & Areia & Valor de $\mathbf{p}$ \\
\hline Consumo de ração & 6611 & 6809 & 0,9955 \\
Ganho de peso & 3007 & 3112 & 0,2589 \\
Conversão alimentar & 2,21 & 2,19 & 0,9305 \\
Viabilidade criatória & 98,33 & 98,33 & 1,0000 \\
Índice de eficiência produtiva & 73,34 & 75,89 & 0,7213 \\
\hline
\end{tabular}

Tabela 4 - Frequência percentual (\%) dos comportamentos manifestados e agrupados por frangos de corte aos 35 dias criados em cama de maravalha e areia (etograma).

\begin{tabular}{lccc}
\hline Comportamentos & Maravalha & Areia & Valor de p \\
\hline Acocorado & 77,60 & 65,20 & \\
Trocando de posição & 3,60 & 3,60 & \\
Comendo & 11,20 & 19,20 & 0,0190 \\
Bebendo & 4,40 & 5,20 & \\
Em pé & 1,20 & 4,80 & \\
Limpando penas & 2,00 & 2,00 & \\
\hline Comportamentos agrupados & & & \\
\hline Ingestivos & 20,72 & 40,00 & $<0,0001$ \\
Repouso & 51,43 & 22,86 & \\
Exploratórios & 11,43 & 19,29 & \\
Conforto & 15,00 & 12,86 & \\
Agressivos & 1,43 & 5,00 & \\
\hline
\end{tabular}

O tipo de material utilizado como cama afetou o comportamento dos frangos de corte $(p<0,05)$. Aves criadas em cama de areia apresentaram menor frequência de 
acocoramento, entretanto mostraram maior frequência de comportamentos de comer, beber e ficar em pé em comparação às aves criadas em cama de maravalha. Ainda, aves criadas em cama de areia apresentaram maior frequência de comportamentos ingestivos, exploratório e agressivos e menor percentual de comportamentos relacionados a repouso e conforto. Em relação à habilidade de locomoção o tipo de cama utilizada influenciou o escore de andadura $(p<0,05)$. Frangos criados em cama de areia apresentaram maiores frequências de aves no escore 0 e menor percentual de aves nos escores 1 e 2 (Tabela 5).

A Tabela 6 mostra os resultados referentes a rendimento de carcaça, cortes comerciais, gordura abdominal, pesos relativos de vísceras comestíveis e órgãos do sistema digestório.

Tabela 5 - Frequência percentual (\%) do escore de andadura em frangos de corte aos 35 dias criados em cama de maravalha e areia (etograma).

\begin{tabular}{lccc}
\hline Escore de andadura & Maravalha & Areia & Valor de $\mathbf{p}$ \\
\hline 0 & 0,00 & 41,67 & \\
1 & 58,33 & 50,00 & 0.0208 \\
2 & 41,67 & 8,33 & \\
\hline
\end{tabular}

Tabela 6 - Rendimento de carcaça e cortes comerciais, peso relativo de vísceras comestíveis e órgãos do sistema digestório de frangos de corte aos 42 dias criados em cama de maravalha e areia.

\begin{tabular}{lccc}
\hline Rendimento de carcaça (\%) & Maravalha & Areia & Valor de $\mathbf{p}$ \\
\hline Carcaça & 74,21 & 74,93 & 0,1057 \\
\hline Rendimento de cortes comerciais (\%) & & & \\
\hline Peito & 34,98 & 37,06 & 0,0338 \\
Coxa & 13,40 & 13,43 & 0,3538 \\
Sobrecoxa & 13,70 & 14,19 & 0,1326 \\
Asas & 12,72 & 12,11 & 0,0493 \\
Gordura abdominal & 1,43 & 1,03 & 0,0370 \\
\hline Vísceras comestíveis (\%) & & & \\
\hline Coração & 0,49 & 0,54 & 0,0806 \\
Fígado & 1,61 & 1,86 & 0,0013 \\
Moela & 1,49 & 1,72 & 0,0132 \\
\hline Órgãos do sistema digestório (\%) & & \\
\hline Proventrículo & 0,34 & 0,33 & 0,5387 \\
Intestino delgado & 3,85 & 4,28 & 0,0313 \\
Pâncreas & 0,26 & 0,27 & 0,2751 \\
\hline
\end{tabular}

O rendimento de carcaça não foi alterado pelo tipo de cama $(p>0,05)$. Entretanto, aves criadas em cama de areia mostraram maior rendimento de peito e menor de asas em comparação às criadas em cama de maravalha $(p<0,05)$. Em relação às vísceras comestíveis, fígado e moela apresentaram maior peso relativo em frangos criados em cama de areia em comparação à maravalha $(p<0,05)$. Resultado semelhante foi observado para o peso relativo do intestino delgado $(p<0,05)$, sem efeito sobre $o$ proventrículo e pâncreas.

\section{DISCUSSÃO}

Os resultados obtidos mostram que é possível equiparar a eficiência da cama de areia com a de maravalha, já que ambas não apresentaram diferença significativa em relação ao desempenho animal. Por outro lado, verificouse maior frequência de comportamentos ativos e menor percentual de comportamentos relacionados a repouso e conforto. Villagrá et al. (2014) mostraram que frangos com menos de 21 dias preferem a maravalha para descansar, entretanto acima dessa idade repousam com mais frequência na areia, além de considerarem a cama de areia a melhor opção para as aves em 
relação aos comportamentos mais ativos. Shields et al., (2005) sugerem que a cama de areia apresenta melhores condições de conforto, além de melhores propriedades isolantes para as aves, mas essa teoria deve ser melhor investigada.

A criação em cama de areia resultou em maior percentual de aves sem problemas locomotores em comparação á cama de maravalha. Nesse sentido, a criação em cama de maravalha ocasionou maior frequência de aves com dificuldades de locomoção. De acordo com Garcês et al., (2013), camas de areia apresentam maior taxa de perda de umidade em comparação á maravalha em função do maior acúmulo de material orgânico grosseiro entre as partículas finas, que aumenta a capacidade de absorver e liberar água. Nesse sentido, a menor retenção de umidade na cama de areia pode contribuir para os menores índices de lesões do aparelho locomotor observados.

Em relação às características da carcaça, aves criadas em cama de areia apresentaram maior rendimento de peito e menor de asas. Ainda, a criação das aves em cama de areia resultou em maior peso relativo de fígado, moela e intestino delgado. Abreu et al. (2011) relataram maior rendimento de peito nos frangos criados em camas de areia em relação aos criados na maravalha. Provavelmente esses resultados podem ser atribuídos à maior atividade das aves nas camas de areia, que proporciona maior desenvolvimento muscular. De acordo com Brito et al. (2016) e Atencio et al. (2010), as partículas de areia ficam retidas por mais tempo na moela, limitando a taxa de passagem dos alimentos, aumentando a absorção de nutrientes pelo intestino delgado e a metabolização dos mesmos pelo fígado.

\section{CONCLUSÃO}

Pode-se concluir que a cama de areia é uma alternativa viável para substituição da maravalha, não interferindo no desempenho, mas melhorando a atividade das aves, além de melhorar o rendimento de peito e aumentar o peso relativo de órgãos do sistema digestório. Os resultados sugerem que aves criadas em cama de areia apresentam melhores condições comportamentais.

\section{REFERÊNCIAS}

ABREU, V. M. N; ABREU, P. G.; COLDEBELLA, A.; JAENISCH, F. R. F; HASSEMER, M. J.; CESTONARO, $T$. Rendimento de carcaça, partes e gordura abdominal de frangos de corte criados em cinco diferentes materiais de cama. In: REUNIÃO ANUAL DA SOCIEDADE BRASILEIRA DE ZOOTECNIA, 48. Anais... Belém, 2011.

ANISUZZAMAN, M.; CHOWDHURY, S. D. Use of four types of litter for rearing broilers. British Poultry Science, v.37, n.3, p.541-545, 1996. https://doi.org/10.1080/00071669608417883

ATENCIO, J. L.; FERNÁNDEZ, J. A.; GERNAT, A. G.; MURILLO, J. G. Effect of pine wood shavings, rice hulls and river bed sand on broiler productivity when used as a litter sources. Int J Poult Sci, v.9, p.240-243, 2010. https://doi.org/10.3923/ijps.2010.240.243

AVILA, V. S. D.; OLIVEIRA, U. D.; FIGUEIREDO, E. A. P. D.; COSTA, C. A. F.; ABREU, V. M. N.; ROSA, P. $S$. Alternative material to replace wood shavings as broiler litter. Revista Brasileira de Zootecnia, v.37, n.2, 273-277, 2008. https://doi.org/10.1590/S1516-

35982008000200013

BILGILI, S. F.; MONTENEGRO, G. I.; HESS, J. B.; ECKMAN, M. K. Sand as litter for rearing broiler chickens. The Journal of Applied Poultry Research, v.8, n.3, p.345-351, 1999a. https://doi.org/10.1093/japr/8.3.345

BILGILI, S. F.; MONTENEGRO, G. I.; HESS, J. B.; ECKMAN, M. K. Live performance, carcass quality, and deboning yields of broilers reared on sand as a litter source. The Journal of Applied Poultry Research, v.8, n.3, p.352-361, 1999b. https://doi.org/10.1093/japr/8.3.352

BILGILI, S. F.; HESS, J. B.; BLAKE, J. P.; MACKLIN, K. S.; SAENMAHAYAK, B.; SIBLEY, J. L. Influence of bedding material on footpad dermatitis in broiler chickens. The Journal of Applied Poultry Research, v.18, n.3, p.583-589, 2009. https://doi.org/10.3382/japr.2009-00023

BIZERAY, D.; ESTEVEZ, I.; LETERRIER, C.; FAURE, J. $M$. Influence of increased environmental complexity on leg condition, performance, and level of fearfulness in broilers. Poultry Science, v.81, n.6, p.767-773, 2002. https://doi.org/10.1093/ps/81.6.767

BONAMIGO, A.; SILVA, C.B.; MOLENTO, C.F. Grau de bem-estar relativo de frangos em diferentes densidades de lotação. Arq. Bras. Med. Vet. Zootec., v.63, n.6, p.1421-1428, 2011. https://doi.org/10.1590/S0102-

09352011000600020

BRITO, D. A. P.; BRITO, D. R. B.; GOMES, A. M. N.; DOS SANTOS CUNHA, A.; SILVA FILHO, U. A.; PINHEIRO, A. A. Desempenho produtivo e rendimento de carcaça de frangos criados em diferentes materiais de cama aviária. Ciência 
Animal Brasileira, v.17, n.2, p.192-197, 2016. https://doi.org/10.1590/1089-6891v17i220736 BUYSE, J. P. C. M.; SIMONS, P. C. M.; BOSHOUWERS, F. M. G.; DECUYPERE, E. Effect of intermittent lighting, light intensity and source on the performance and welfare of broilers. World's Poultry Science Journal, v.52, n.02, 121-130, 1996. https://doi.org/10.1079/WPS19960012

CARVALHO, T. M. R. D.; MOURA, D. J. D.; SOUZA, Z. M. D.; SOUZA, G. S. D.; BUENO, L. G. D. F. Qualidade da cama e do ar em diferentes condições de alojamento de frangos de corte. Pesquisa Agropecuária Brasileira, 2011. https://doi.org/10.1590/S0100-

204X2011000400003

CORR, S. A.; GENTLE, M. J.; MCCORQUODALE, C. C.; BENNETT, D. The effect of morphology on walking ability in the modern broiler: a gait analysis study. Animal Welfare, v.12, n.2, p.159171, 2003.

GARCÊS, A.; AFONSO, S. M. S.; CHILUNDO, A.; JAIROCE, C. T. S. Evaluation of different litter materials for broiler production in a hot and humid environment: 1 . Litter characteristics and quality. The Journal of Applied Poultry Research, v.22, n.2, p.168-176, 2013. https://doi.org/10.3382/japr.2012-00547

HAVENSTEIN, G. B.; FERKET, P. R.; QURESHI, M. A. Growth, livability, and feed conversion of 1957 versus 2001 broilers when fed representative 1957 and 2001 broiler diets. Poultry Science, v.82, n.10, p.1500-1508, 2013. https://doi.org/10.1093/ps/82.10.1500

JONES, T. A.; DONNELLY, C. A.; DAWKINS, M. S. Environmental and management factors affecting the welfare of chickens on commercial farms in the United Kingdom and Denmark stocked at five densities. Poultry Science, v.84, n.8, p.1155-1165, 2005. https://doi.org/10.1093/ps/84.8.1155

LIEN, R. J.; CONNER, D. E.; BILGILI, S. F. The use of recycled paper chips as litter material for rearing broiler chickens. Poultry Science, v.71, n.1, p.8187, 1992. https://doi.org/10.3382/ps.0710081 MILES, D. M.; ROWE, D. E.; CATHCART, T. C. Litter ammonia generation: moisture content and organic versus inorganic bedding materials. Poultry Science, v.90, n.6, p.11621169, 2011. https://doi.org/10.3382/ps.201001113

NEME, R.; SAKOMURA, N. K.; DE OLIVEIRA, M. D. S.; LONGO, F. A.; FIGUEIREDO, A. N. Adição de gesso agrícola em três tipos de cama de aviário na fixação de nitrogênio e no desempenho de frango de corte. Ciência Rural, v.30, n.4, p.687-
692, 2000. https://doi.org/10.1590/S010384782000000400022

PAGANINI, F. J.; MENDES, A. A. Manejo de cama. Produção de Frangos de Corte. Brasil: Facta, 2004. p. 108-115.

PICOLI, K. P. Avaliação de sistemas de produção de frangos de corte no pasto. 2004. Dissertação (Mestrado em Agroecossistemas) - Universidade Federal de Santa Catarina, Florianópolis, 2004.

ROSTAGNO, H. S.; ALBINO, L. F. T.; DONZELE, J. L.; GOMES, P. C.; OLIVEIRA, R. F.; LOPES, D. C.; EUCLIDES, P. F. Tabelas brasileiras para aves e suínos. Composição de Alimentos e Exigências Nutricionais. 3. ed. Viçosa: UFV, 2011.

SHIELDS, S. J.; GARNER, J. P.; MENCH, J. A. Effect of sand and wood-shavings bedding on the behavior of broiler chickens. Poultry Science, v.84, n.12, p.1816-1824, 2005. https://doi.org/10.1093/ps/84.12.1816

VILLAGRÁ, A.; OLIVAS, I.; ALTHAUS, R. L.; GÓMEZ, E. A.; LAINEZ, M.; TORRES, A. G. Behavior of broiler chickens in four different substrates: a choice test. Revista Brasileira de Ciência Avícola, v.16, n.1, p.67-75, 2014. https://doi.org/10.1590/S1516-

635X2014000100010

WEEKS, C. A.; DANBURY, T. D.; DAVIES, H. C.; HUNT, P.; KESTIN, S. C. The behaviour of broiler chickens and its modification by lameness. Applied Animal Behaviour Science, v.67, n.1, p.111-125, 2000. https://doi.org/10.1016/S0168-1591(99)00102-1

Recebido para publicação em 25/07/2016 Revisado em 15/08/2016

Aceito em 22/08/2016 